\title{
Prognostic and predictive impact of creatine kinase level in non-small cell lung cancer treated with tyrosine kinase inhibitors
}

\author{
Yu Jiang ${ }^{1,2,3,4,5 \#}$, Zixuan Su${ }^{1,2,3,4,5 \#}$, Yuechun Lin ${ }^{1,2,3,4,5 \#}$, Yaming Xiong ${ }^{1,2,3,4 \#}$, Caichen Li $^{1,2,3,4}$, Jianfu Li ${ }^{1,2,3,4}$, \\ Runchen Wang ${ }^{1,2,3,4,5}$, Ran Zhong ${ }^{1,2,3,4}$, Bo Cheng ${ }^{1,2,3,4}$, Jianxing He $\mathbf{e}^{1,2,3,4}$, Zhanhong Xie ${ }^{2,3,4,6}$, Wenhua Liang ${ }^{1,2,3,4}$ \\ ${ }^{1}$ Department of Thoracic Surgery and Oncology, the First Affiliated Hospital of Guangzhou Medical University, Guangzhou, China; ${ }^{2}$ State Key \\ Laboratory of Respiratory Disease, Guangzhou, China; ${ }^{3}$ National Clinical Research Center for Respiratory Disease, Guangzhou, China; ${ }^{4}$ Guangzhou \\ Institute of Respiratory Health, Guangzhou, China; ${ }^{5}$ Nanshan School, Guangzhou Medical University, Guangzhou, China; ${ }^{6}$ Department of \\ Respiratory Medicine, the First Affiliated Hospital of Guangzhou Medical University, Guangzhou, China \\ Contributions: (I) Conception and design: Y Jiang, Z Xie, W Liang; (II) Administrative support: J He, Z Xie, W Liang; (III) Provision of study \\ materials or patients: All authors; (IV) Collection and assembly of data: Y Jiang, Z Su, C Li, J Li, R Zhong; (V) Data analysis and interpretation: Y \\ Jiang, Z Su, Y Lin, Y Xiong, R Wang, B Cheng; (VI) Manuscript writing: All authors; (VII) Final approval of manuscript: All authors. \\ "These authors contributed equally to this work. \\ Correspondence to: Wenhua Liang, MD. Department of Thoracic Oncology, the First Affiliated Hospital of Guangzhou Medical University, Guangzhou, \\ China; China State Key Laboratory of Respiratory Disease \& National Clinical Research Center for Respiratory Disease, Guangzhou, China. Email: \\ liangwh1987@163.com; Zhanhong Xie, MD. Department of Respiratory Medicine, the First Affiliated Hospital of Guangzhou Medical University, \\ Guangzhou, China; State Key Laboratory of Respiratory Disease \& National Clinical Research Center for Respiratory Disease, Guangzhou, China. \\ Email: 13556184135@163.com.
}

Background: The use of tyrosine kinase inhibitors (TKIs) is associated with incident creatine kinase (CK) elevation in the treatment of advanced non-small cell lung cancer (NSCLC) patients. However, whether higher CK levels are associated with better antitumor responses or survival remains to be explored. We intend to investigate the clinical correlation between CK levels and TKI efficacy in advanced NSCLC.

Methods: In this retrospective study, we enrolled 135 patients with stage IV NSCLC receiving TKI-based therapy in our center between June 2012 to July 2020. CK levels were monitored from the initiation of TKI medication and during the administration period. An X-tile analysis provided the optimal cutoff point for higher baseline CK. Patients were identified and grouped according to their baseline CK level and fold changes during TKI therapy. The primary endpoints included progression-free survival (PFS) and overall survival (OS), and the objective response rate (ORR) was calculated as the secondary endpoint.

Results: Among the 135 patients included in our study, those with higher baseline CK levels $(\geq 70$ U/L) had favorable PFS (15.2 vs. 8.8 months; $\mathrm{P}=0.028$ ), while patients with significantly elevated CK (the highest $\mathrm{CK}$ value/baseline $\mathrm{CK}$ value $\geq 2$ times) appeared to gain better PFS (14.6 vs. 10.0 months; $\mathrm{P}=0.139$ ). The overall ORR was $67.4 \%$. Patients with higher baseline CK levels had numerically higher ORR (74.6\% vs. $60.3 \% ; \mathrm{P}=0.076)$. Similarly, patients with significant CK elevation had a superior 4-month PFS rate $(77.6 \%$ vs. $59.7 \% ; \mathrm{P}=0.029)$. Results from the subgroup analyses were identical to the overall ones. For patients with higher baseline CK levels, those experiencing significant CK elevation had prolonged PFS (17.2 vs. 14.2 months; $\mathrm{P}=0.038$ ); a same trend was obtained from the lower baseline CK group ( $<70 \mathrm{U} / \mathrm{L})(9.4$ vs. 7.9 months; $\mathrm{P}=0.038)$. In multivariable analysis, higher baseline $\mathrm{CK}$ level and significant CK elevation remained statistically associated with PFS, with hazard ratios of 0.48 and 0.59 , respectively.

Conclusions: Both higher baseline CK levels and significant CK elevation after treatment were correlated with prolonged PFS in NSCLC treated with TKIs, suggesting the potential prognostic and predictive impact of CK level on these patients.

Keywords: Tyrosine kinase inhibitors (TKIs); creatine kinase (CK); non-small cell lung cancer (NSCLC); prognosis 
Submitted Jul 26, 2021. Accepted for publication Sep 09, 2021.

doi: $10.21037 /$ tlcr-21-600

View this article at: https://dx.doi.org/10.21037/tlcr-21-600

\section{Introduction}

Lung cancer is the second most commonly diagnosed cancer worldwide and the leading cause of global cancer death in 2020. In China, it ranks first in terms of cancer incidence and mortality, and is responsible for over 815,563 new cases and 714,699 deaths, representing approximately one in $5(17.9 \%)$ cancer diagnosed and a quarter of $(23.8 \%)$ cancer deaths in 2020 (1). Non-small cell lung cancer (NSCLC) accounts for $85 \%$ of lung cancer and over $50 \%$ of NSCLC patients were diagnosed at an advanced stage (2).

In the last decade, the discovery of specific oncogenic driver mutations in lung cancer represents valid therapeutic targets and enables individualized targeted treatment that translates into clinically benefit. Specifically, the most common activating mutations in adenocarcinoma include epidermal growth factor receptor (EGFR), which occurs in up to $40 \%$ of Asian patients, rearrangements of anaplastic lymphoma kinase (ALK), c-ROS oncogene 1 (ROS-1), and several additional mutations (3-5). Novel treatment options, such as tyrosine kinase inhibitors (TKIs), have been recommended as first-line therapy for naive and recurrent advanced NSCLC, showing favorable clinical outcomes, enhanced response rate, and greater potency (6). These include gefitinib, erlotinib, afatinib, and osimertinib for EGFR-mutated NSCLC, alectinib, crizotinib, ceritinib, brigatinib and lorlatinib for ALK-rearranged NSCLC, and crizotinib for ROS-1 mutant NSCLC (7). TKIs are small molecules that interfere with the kinase activity by competing against ATP binding sites of the catalytic domain in specific oncogenic tyrosine kinases (8). However, TKIs use in cancer treatment can sometimes result in a certain degree of side effects that affect multiple organs, such as diarrhea, acneiform rash, paronychia, stomatitis, abnormal liver function, and gastrointestinal events (9-11).

CK elevation is one of the recognized adverse events, which may be attributed to the inhibition of both ABL and AMPactivated protein kinase, occurring in approximately one-third of patients who received TKIs treatment for solid tumors (12-14). Increasing evidence suggested that serum CK levels may be a potential predictor of development and progression in a variety of cancers, including breast cancer (15). In addition, CK inactivation has been closely related to the deterioration of oral squamous cell carcinoma (SCC) (16). It is therefore critical that CK levels during TKI therapy be appropriately monitored and managed, as they may provide biochemical indicators of clinical outcome and aid in drug dosage adjustment if necessary. Nonetheless, no clinical studies have been conducted so far to determine whether CK levels are correlated with better clinical outcomes for advanced NSCLC patients treated with TKIs.

In light of those data, we conducted a preliminary analysis in a real-world setting to investigate whether plasma CK levels could be used as independent factors for predicting the prognosis and further identifying the dominant population with the optimal clinical outcome. We present the following article in accordance with the REMARK reporting checklist (available at https://dx.doi. org/10.21037/tlcr-21-600).

\section{Methods}

\section{Study population}

This single-center, retrospective cohort study was carried out at the First Affiliated Hospital of Guangzhou Medical University. We reviewed the medical records of all adult patients with naive or recurrent advanced NSCLC treated with TKI monotherapy or TKI-based combined therapy between June 2012 to July 2020. Patients with a histologically confirmed diagnosis of NSCLC, treatment with one of the common TKIs (osimertinib, erlotinib, gefitinib, icotinib, crizotinib, bevacizumab, afatinib, ceritinib, brigatinib, apatinib, ensartinib, anlotinib, alectinib, cetuximab, or vemurafenib), and available CK levels before and periodically during treatment and follow-up of at least 14 days were included in this study, while those known to have any pre-existing disease that can cause a pathological elevation of CK were excluded, such as myocardial infarction (MI), myopathies, myositis, dystrophies, hyper/ hypothyroidism, or renal insufficiency. Also, patients whose CK status could not be determined reliably were excluded as well.

All procedures performed in this study were in accordance with the Declaration of Helsinki (as revised in 2013). The study was approved by the Institutional Review Board of the 
First Affiliated Hospital of Guangzhou Medical University (No. KLS-2020), allowing the extracted data to be used for research purposes. Written informed consents were waived by the ethical committee since this is a retrospective study.

\section{Data collection}

Baseline clinical data were extracted from electronic patient records, including relevant demographic data [age, gender, and Eastern Cooperative Oncology Group (ECOG) performance status (PS)], cancer-related data (pathology, tumor stage, and mutation profile), treatment data [CK value, TKI type, generation, treatment line, evaluation interval, combination therapy, adverse event (AE), and discontinuation], and outcome data (date of death or last follow-up).

As is commonly seen clinically, advanced NSCLC patients received different therapies, such as TKI monotherapy, or TKI-based combination with chemotherapy, immune checkpoint inhibitors (ICIs), or another TKI. Although mechanisms of TKI-related CK elevation have been proposed, it remains an area of ongoing research. Therefore, we collected and reported various types of TKI agents and classes of therapy that have not yet been known to be associated with CK elevation. Management of TKIs and evaluation of efficacy were determined by each researcher. In this retrospective study, the administration of different TKIs was affected by previous clinical practice and updated clinical indications for TKIs during the study.

Collected CK data included the history of cardiovascular events, muscular disorders and renal abnormality, relevant myocardial enzymes status, history of surgery within 5 days before TKI treatment, plasma CK levels before and periodically during TKI therapy, date of first CK elevation, and the highest serum CK value. To calculate fold changes, CK status was assessed using venous blood biochemistry tests within the first 2 weeks from the initiation of TKI medication, every 4-8 weeks thereafter, and whenever disease progression occurred during the treatment period. The diagnosis and severity of AEs were appraised under clinical manifestations, biological examinations, and imaging data, and were classified and graded according to the Common Terminology Criteria for Adverse Events (CTCAE) version 4.0.

The antitumor response was determined based on the results of clinical examinations, computed tomography (CT) scans and/or lymph node biopsy, and the date of death was obtained via a follow-up visit or telephone inquiry.
Chest CT scans were performed bimonthly during TKI treatment, following the Response Evaluation Criteria for Solid Tumors (RECIST) ver. 1.1.

\section{Statistical analysis}

To summarize the baseline patient characteristics, means with standard deviation and medians with interquartile range (IQR) were used for normally and non-normally continuous distributed variables, respectively. Numbers reported with percentages were used for categorical variables. As an exploratory analysis of CK, clinical characteristics were compared using Student's $t$-test or the Mann-Whitney U-test for continuous variables, and Pearson's chi-square test or Fisher's exact test for categorical variables. A second chi-square analysis was performed to determine the relationship between the baseline CK level and the highest CK level.

Primary outcomes for this retrospective study were progression-free survival (PFS) and overall survival (OS), and the secondary outcome was objective response rate (ORR), namely the sum of partial response (PR) and complete response (CR), and also the 4-month PFS rate. PFS and OS were estimated using the Kaplan-Meier method. We defined PFS as the time from the day of TKI initiation to the earliest signs of disease progression or death. OS was calculated from the first day of TKIbased treatment to death or the last follow-up visit. Survival curves from 4 groups were compared using a two-sided log-rank test. After checking the proportional assumptions, the Cox proportional-hazards model was adopted to estimate the hazards ratios (HRs) for PFS and OS. Each baseline parameter was first evaluated in the univariate model, and significant variables were then included in the multivariate model to further identified the independent predictors of the optimal clinical outcome in advanced NSCLC patients. In addition, subgroup analyses were performed to estimate the differences in PFS across all prespecified subgroups, stratified by age, gender, TKI type, and treatment received. Also, the ORR was calculated for each subgroup. Differences with $\mathrm{P}$ value $<0.05$ were considered statistically significant. The outcome-based cut-point optimization was performed using X-tile software (version 3.6.1, Yale University School of Medicine, New Haven, CT, USA) (17). All the other statistical analyses were performed using IBM SPSS Statistics software (version 26.0), R software (version 4.1.0), and GraphPad Prism software (version 9.00). 


\section{Results}

The clinical features of 135 patients with stage IV NSCLC, stratified by their baseline CK values and fold changes during TKI therapy, were outlined in Table 1 . The median follow-up time was 19.1 months (range, 0.5-64.9 months). The following molecular alterations were identified in our study population, with 65 (48.1\%) for EGFR, 11 (8.1\%) for ALK rearrangement, 5 (3.7\%) for ROS1, 4 (3.0\%) for cMET, $1(0.7 \%)$ for BRAF, and $1(0.7 \%)$ for HER2. Patients in the study received 15 different TKIs, and the number of patients for each medication was shown in Table 2. Regarding TKI type, 74 (54.8\%), 31 $(23.0 \%)$, and $30(22.2 \%)$ patients were treated with firstgeneration or second-generation EGFR-TKI, thirdgeneration EGFR-TKI, and ALK/ROS1-TKI. Among 135 patients, $115(85.2 \%)$ underwent monotherapy, while the remaining $20(14.8 \%)$ received TKI-based combinations with concomitant use of other anticancer medication, of which $6(4.4 \%), 7(5.2 \%)$, and 8 (5.9\%) were treated with chemotherapy, immune checkpoint inhibitors (ICIs) and another TKI, respectively.

The median CK value of all patients was $69 \mathrm{U} / \mathrm{L}$, and the optimal cutoff value for a higher baseline CK level was $\geq 70 \mathrm{U} / \mathrm{L}$. Of 135 patients enrolled in this study, significantly elevated CK (the highest CK value/baseline CK value $\geq 2$ times) occurred in $58(43 \%)$ patients. Notably, all patients with elevated CK were reported asymptomatic and no further management like dose reduction was performed for these patients. Comparisons of clinical parameters between groups were also shown in Table 1. Men were more likely to have higher baseline CK levels than women [40/69 (58.0\%) vs. 27/66 (40.9\%); $\mathrm{P}=0.047]$. What's more, the incidence of significant CK elevation was higher in male than female [36/69 (52.2\%) vs. $22 / 66$ (33.3\%); $\mathrm{P}=0.027$ ]. Other than that, there was no statistical difference among other demographic factors.

To examine the prognostic impact of CK, all 135 patients were firstly divided into two groups based on the median value of baseline CK, including a higher baseline CK group $(\geq 70 \mathrm{U} / \mathrm{L})$ and a lower baseline CK group ( $<70 \mathrm{U} / \mathrm{L})$. In addition, to investigate the predictive impact of $\mathrm{CK}$, we divided 135 patients into two groups according to the fold changes of CK after treatment. The Kaplan-Meier survival curves for higher and lower baseline CK groups were shown in Figure 1A. A longer median PFS was observed in patients with higher baseline CK compared to those with lower baseline CK (median PFS: 15.2 vs. 8.8 months; $\mathrm{P}=0.028$ ).
A similar trend was obtained from responders with and without significant CK elevation (median PFS: 14.6 vs. 10.0 months; $\mathrm{P}=0.139$ ), as shown in Figure $1 B$. Regarding OS, there was no significant difference between these groups (Figure S1). Results of the ORRs were concordant with the PFS ones. The overall ORR was $67.4 \%$. Patients with higher baseline CK levels had better ORR than the lower baseline CK group, but there was no significant difference between the two groups $(74.6 \%$ vs. $60.3 \%$; $\mathrm{P}=0.076)$. Likewise, ORRs were higher among patients experiencing significant CK elevation compared with those without (77.6\% vs. $59.7 \%$; $\mathrm{P}=0.029)$. In subgroup analyses, owing to the limited number of patients in some subgroups, more attention should be paid to the effect estimates instead of statistical significance. The clinical efficacy for patients experiencing higher CK levels and significant CK elevation were consistently favorable in most of the prespecified subgroups (Figures S2,S3).

To further determine whether higher CK levels were correlated with better efficacy of TKIs, we further divided all patients into four subgroups based on both baseline CK level and CK change during TKI therapy, namely, higher baseline CK group with significantly elevated CK $(n=27)$; higher baseline CK group without significantly elevated CK $(n=40)$; lower baseline CK group with significantly elevated CK (n=31); lower baseline CK group without significantly elevated CK $(n=37)$. In terms of survival or response, the median PFS for the four groups were 17.2, 14.2, 9.4, and 7.9 months $(\mathrm{P}=0.038)$, respectively. The Kaplan-Meier curves of PFS in these groups were presented in Figure 2.

The chi-square test showed no significant effect on baseline CK values and the highest CK level $\left(\chi^{2}=0.385\right.$; $\mathrm{P}=0.535)$, indicating that baseline CK level did not affect the highest CK level during the therapy. Also, Cox multivariable regression analysis revealed that higher baseline CK level and significant CK elevation could act as independent factors for a better prognosis for TKItreated NSCLC patients, as shown in Table 3. Statistically significant differences were observed in PFS, indicating a $52 \%$ and $41 \%$ reduction in the risk of progression or death in patients with higher baseline CK [HR $=0.48$ (95\% CI: 0.28-0.81); $\mathrm{P}=0.006$ ] and with significant CK elevation [HR $=0.59$ (95\% CI: $0.35-0.99) ; \mathrm{P}=0.047$ ].

\section{Discussion}

With the extensive application of TKIs profiling in clinical practice, the clinical relevance of TKI-related CK elevation 
Table 1 Baseline clinical characteristics of the 135 patients with advanced NSCLC treated with TKIs, stratified by baseline CK level and CK change during treatment

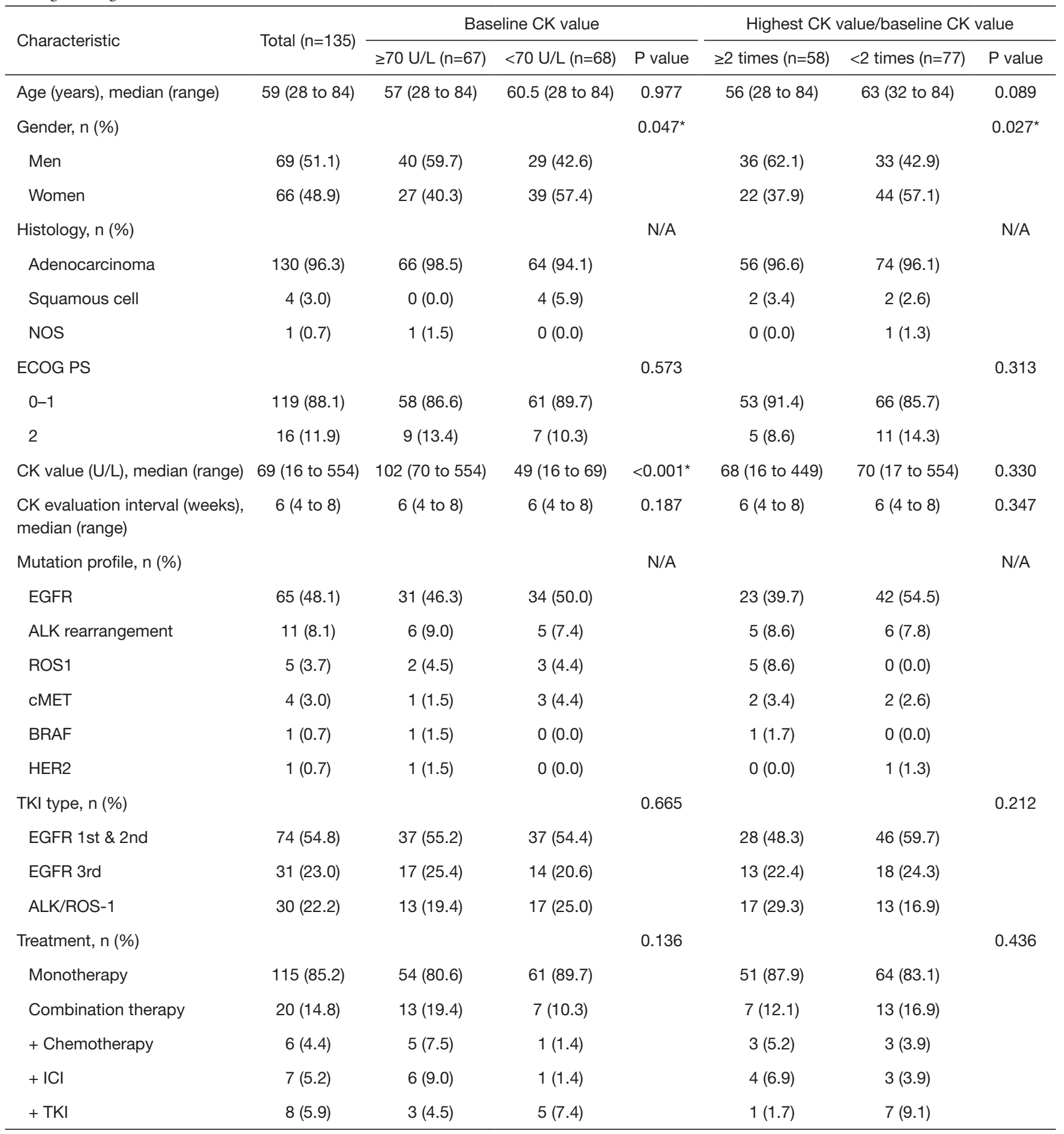

Table 1 (continued) 
Table 1 (continued)

\begin{tabular}{|c|c|c|c|c|c|c|c|}
\hline Characteristic & Total $(n=135)$ & \multicolumn{3}{|c|}{ Baseline CK value } & \multicolumn{3}{|c|}{ Highest CK value/baseline CK value } \\
\hline Adverse event, n (\%) & & & & $\mathrm{N} / \mathrm{A}$ & & & $\mathrm{N} / \mathrm{A}$ \\
\hline Liver function abnormalities & $5(3.7)$ & $3(4.5)$ & $2(2.9)$ & & $2(3.4)$ & $3(3.9)$ & \\
\hline Diarrhea & $15(11.1)$ & $8(11.9)$ & $7(10.3)$ & & $11(19.0)$ & $4(5.2)$ & \\
\hline Paronychia & $14(10.4)$ & $8(11.9)$ & $6(8.8)$ & & $9(15.5)$ & $5(6.5)$ & \\
\hline Discontinuation, $\mathrm{n}(\%)$ & & & & $\mathrm{N} / \mathrm{A}$ & & & $\mathrm{N} / \mathrm{A}$ \\
\hline $\begin{array}{l}\text { Discontinuation due to } \mathrm{CK} \\
\text { elevation }\end{array}$ & $0(0.0)$ & $0(0.0)$ & $0(0.0)$ & & $0(0.0)$ & $0(0.0)$ & \\
\hline
\end{tabular}

Table 2 Number of patients treated with each TKI drug in the study population, stratified by both baseline CK level and CK change during TKI therapy

\begin{tabular}{|c|c|c|c|c|c|}
\hline \multirow{2}{*}{ TKI name } & \multirow{2}{*}{ Total $(n=135)$} & \multicolumn{2}{|c|}{ Baseline CK value } & \multicolumn{2}{|c|}{ Highest CK value/baseline CK value } \\
\hline & & $\geq 70 \mathrm{U} / \mathrm{L}(\mathrm{n}=67)$ & $<70 \mathrm{U} / \mathrm{L}(\mathrm{n}=68)$ & $\geq 2$ times $(n=58)$ & $<2$ times $(\mathrm{n}=77)$ \\
\hline Erlotinib & 26 & 13 & 13 & 9 & 17 \\
\hline Gefitinib & 23 & 12 & 11 & 10 & 13 \\
\hline Crizotinib & 19 & 8 & 11 & 11 & 8 \\
\hline Bevacizumab & 6 & 6 & 0 & 4 & 2 \\
\hline Afatinib & 5 & 3 & 2 & 1 & 4 \\
\hline Ceritinib & 5 & 2 & 3 & 3 & 2 \\
\hline Ensartinib & 2 & 1 & 1 & 1 & 1 \\
\hline Anlotinib & 1 & 0 & 1 & 0 & 1 \\
\hline Alectinib & 1 & 0 & 1 & 0 & 1 \\
\hline Cetuximab & 1 & 0 & 1 & 0 & 1 \\
\hline Vemurafenib & 1 & 1 & 0 & 1 & 0 \\
\hline
\end{tabular}

CK, creatine kinase; TKI, tyrosine kinase inhibitor. 
A
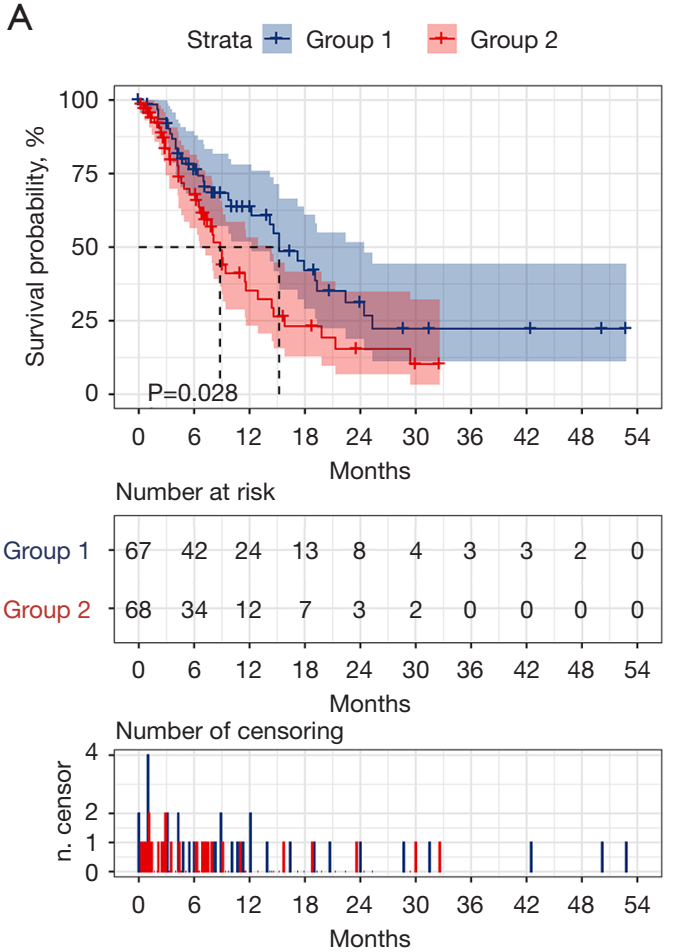

B

Strata + Group 3 + Group 4
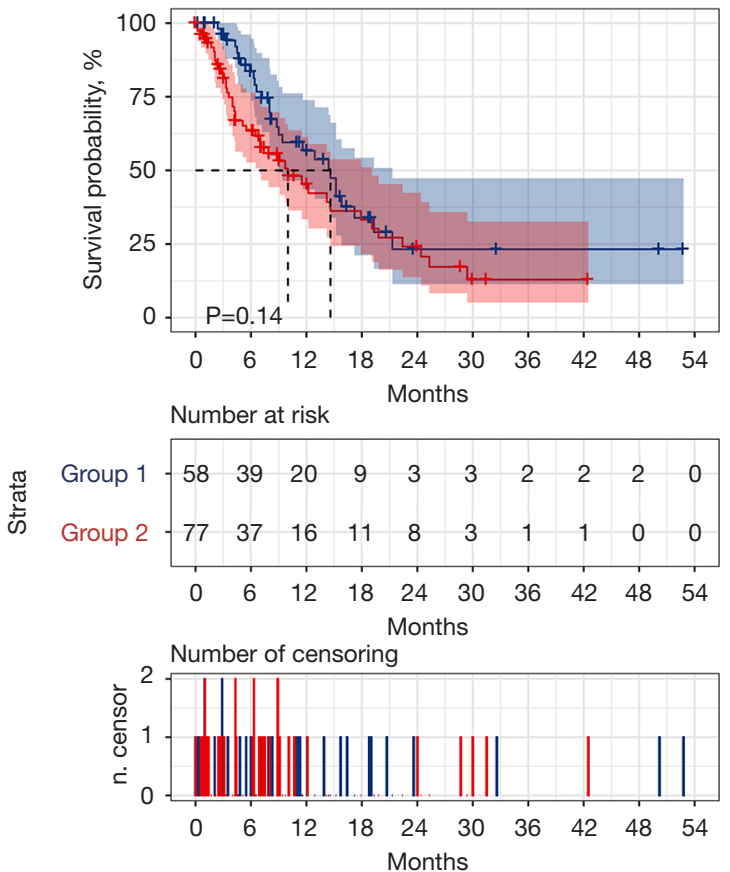

Figure 1 Kaplan-Meier curves for progression-free survival in advanced NSCLC patients treated with TKIs, stratified by (A) baseline CK level, and (B) CK change during TKI therapy. Group 1: higher baseline CK group (n=67); Group 2: lower baseline CK group (n=68); Group 3: patients with significantly elevated CK during TKI therapy (n=58); Group 4: patients without significantly elevated CK during TKI therapy $(\mathrm{n}=77)$. CK, creatine kinase; NSCLC, non-small cell lung cancer; TKI, tyrosine kinase inhibitor.

has been increasingly recognized. To date, only a few studies have reported on the association between adverse effects and the effectiveness of TKI therapy, and whether such a correlation exists for CK expression and TKI efficacy in NSCLC patients remains unknown. In this retrospective cohort study, we analyzed the prognoses of patients with different baseline CK levels and CK changes. We found that patients with higher CK baseline levels and significant CK elevation were more likely to have better clinical efficacy and survival status, and it was further supported by multivariable analyses and subgroup analyses. To the best of our knowledge, our study is the first prognostic analysis to simultaneously investigate both the prognostic and predictive impact of CK level.

$\mathrm{CK}$ is an enzyme expressed by various cells and tissues (18). It is essential for energy homeostasis in tissues with high and fluctuating energy demands by connecting ATP production sites with consumption sites and buffering the intracellular ATP/ADP ratio, particularly in cardiac and skeletal muscles (19). Several studies have reported on
CK dysregulation in different malignancies, demonstrating that with the progression of malignant tumors, the activity of CK in muscles gradually decreases and it cannot even be detected in the final stage of dedifferentiation (20). Namely, low levels of CK may represent a sign of tumorigenesis or deterioration. The prevalence of CK elevation observed in this study is similar to that of a prospective study involving 155 patients with solid tumors treated with different types of TKIs, revealing a significant association with the incidence of myalgias (14). For patients experiencing TKI therapy, skeletal muscle cells may share similar tyrosinekinase pathways with lung cancer, and these pathways may be inhibited simultaneously. Therefore, drug concentrations with antineoplastic effectiveness could also lead to CK elevation at the same time.

The underlying pathophysiological mechanisms underlying the observed higher CK levels and better prognosis in TKI-treated NSCLC patients were unknown. This interaction could either be causal or suggestive of a potential mechanism that is correlated with both 


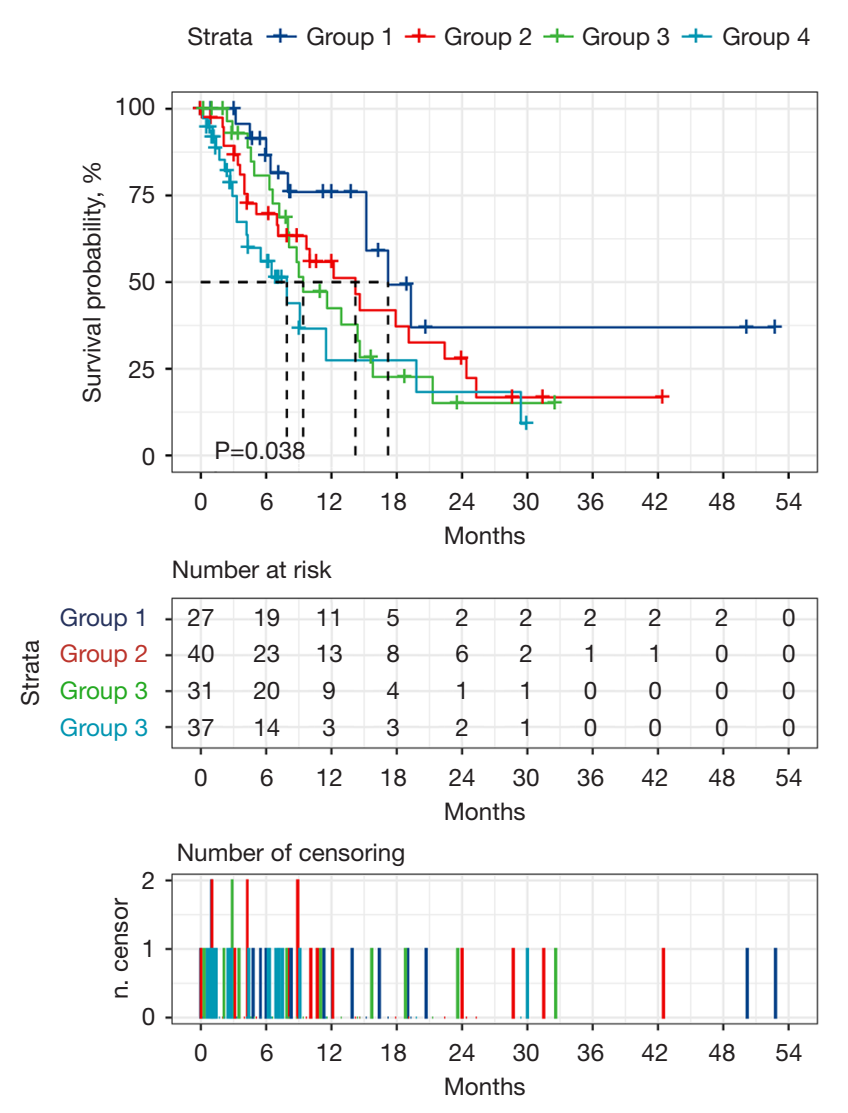

Figure 2 Kaplan-Meier curves for progression-free survival in advanced NSCLC patients treated with TKIs, stratified by both baseline CK level and CK change during TKI therapy. Group 1: higher baseline CK group with significantly elevated CK ( $n=27)$; Group 2: higher baseline CK group without significantly elevated CK (n=40); Group 3: lower baseline CK group with significantly elevated CK ( $n=31)$; Group 4: lower baseline CK group without significantly elevated CK $(n=37)$. CK, creatine kinase; NSCLC, non-small cell lung cancer; TKI, tyrosine kinase inhibitor.

immunological cross-reactivity and anti-tumor response. Previous studies have proven that $\mathrm{CK}$ was considered to be an auxiliary indicator reflecting the status of immune response, including adaptive immune response and innate immune response (21). Also, the improved prognosis was concurrent with higher CK levels in TKI-treated NSCLC patients, which represented the systemic immune response to cancer and further supported the potential effect of autoimmunity. Hence, we hypothesize that CK may play a role in immune response and serum CK levels may reflect the status of autoimmunity of TKI-treated patients to some extent. In this process, TKIs could stimulate the immune system to attack cancer cells, meanwhile, it may lead to a series of autoimmune responses, as the side effect of the successful reversal of cancer occurrence, progression, and metastasis. For instance, dermatomyositis and polymyositis have been described as immune-related adverse events (irAEs) (22-24). Prior researches showing a correlation of irAEs with superior clinical response have substantiated that antigens shared between malignancy and normal tissues might be responsible for this association $(25,26)$. Nevertheless, whether NSCLC cells share antigens with normal tissues influenced by irAEs remains to be explored. Some investigators have observed an improved OS in TKI-treated patients who develop immunerelated hypothyroidism compared with those without hypothyroidism (27). Identical conclusions were obtained in our multivariable analyses, showing a prolonged PFS in patients with higher CK levels, which may attribute to immune-mediated myocyte-damaging effects.

Monitoring serum CK levels before and periodically during TKI therapy has superiority in terms of convenience, cost-effectiveness, and less invasiveness. As reported, our findings revealed the prognostic and predictive role of CK for TKI-treated patients, and it is likely to help physicians perform personalized treatment and interpret the contribution of CK to survival differences in clinical practice. Although the underlying mechanism was not fully understood, its clinical significance could not be overlooked. It is recommended that serum CK profiling should be performed as early as possible for patients treated with TKIs, to further identify the related prognostic indicators and implement necessary intervention and individualized treatment as early as possible. Also, previous studies have provided clinical algorithms in respect of CK management for patients receiving immunotherapy (28). Even so, further validation studies with a large sample size are required to verify its feasibility on NSCLC patients and promote the establishment of optimal administration for TKIs.

The outcomes of our study were affected by several limitations. The main limitation in our analysis was the small sample size, leading to a relatively low explanatory power in survival analyses when evaluating patients with different baseline CK levels as well as different CK changes separately. For one thing, we could not include all potential confounders in our multivariable analysis due to the limited number of available covariates in the study population. For another, the follow-up time was not sufficiently long to further figure out the dominant population of the optimal efficacy. Also, whether the cutoff value calculated 
Table 3 Univariate and multivariate analysis of PFS in advanced NSCLC patients treated with TKIs

\begin{tabular}{|c|c|c|c|c|}
\hline Variable & \multicolumn{2}{|c|}{ Univariate } & \multicolumn{2}{|c|}{ Multivariate } \\
\hline Age & $0.99(0.97-1.01)$ & 0.471 & $0.99(0.96-1.02)$ & 0.393 \\
\hline Gender (female vs. male) & $1.21(0.76-1.94)$ & 0.428 & $1.01(0.60-1.68)$ & 0.985 \\
\hline ECOG PS (2 vs. 0-1) & $0.51(0.20-1.27)$ & 0.146 & $0.71(0.24-2.08)$ & 0.528 \\
\hline EGFR 3rd (EGFR 3rd vs. EGFR 1st \& 2nd) & $1.59(0.91-2.77)$ & 0.103 & $1.68(0.94-3.00)$ & 0.078 \\
\hline ALK/ROS-1 (ALK/ROS-1 vs. EGFR 1st \& 2nd) & $1.03(0.55-1.93)$ & 0.935 & $0.92(0.47-1.80)$ & 0.803 \\
\hline Combination (yes vs. no) & $1.10(0.56-2.16)$ & 0.774 & $1.22(0.60-2.47)$ & 0.582 \\
\hline Baseline $\mathrm{CK} \geq 70 \mathrm{U} / \mathrm{L}$ (yes vs. no) & $0.59(0.37-0.95)$ & $0.030^{*}$ & $0.48(0.28-0.81)$ & $0.006^{*}$ \\
\hline
\end{tabular}

*, P<0.05. ALK, anaplastic lymphoma kinase; Cl, confidence interval; CK, creatine kinase; ECOG PS, Eastern Cooperative Oncology Group performance status; EGFR, epidermal growth factor receptor; HR, hazard ratio; NSCLC, non-small cell lung cancer; PFS, progression-free survival; ROS-1, c-ROS oncogene 1; TKI, tyrosine kinase inhibitor.

from limited participants could be applied to other TKItreated NSCLC patients remains to be verified. In addition, considering the retrospective nature of our study, we cannot fully differentiate the prognostic and predictive impact of CK level on patients. ORRs have suggested a predictive effect on patients treated with TKIs while the lack of CK monitoring for patients receiving general radiotherapy and chemotherapy hampered exploring whether higher baseline CK level could be regarded as a prognostic indicator. Further detailed prospective analyses are currently in progress. Although we proposed that higher serum CK levels might inhibit the occurrence and development of lung cancer, there has been no definite mechanism to elucidate this clinical phenomenon, and the findings should, in this respect, be interpreted with caution. Therefore, future prospective studies with larger sample sizes and a more rigorous design are highly warranted to draw definitive conclusions.

\section{Conclusions}

We found that both higher baseline CK level and significant CK elevation after treatment were associated with improved PFS, suggesting that CK might be an auxiliary prognostic and predictive factor of durable efficacy in patients treated with TKIs. Additional large-scale randomized clinical trials and fundamental studies should attach more importance to investigating the interaction between CK elevation and clinical efficacy of TKIs, assessing the risk-benefit profile to better guide clinical treatment.

\section{Acknowledgments}

The authors would like to thank Ms. Xueqing Liang for her technical assistance. Also, we are grateful to the editors and anonymous reviewers for their insightful comments and suggestions that helped us improve the quality of our paper. Funding: This work was supported by the National Key R\&D Program of China (2016YFC0905400), China National Science Foundation (81871893, 81501996), Key Project of Guangzhou Scientific Research Project (201804020030), High-level University Construction Project of Guangzhou Medical University (20182737, 201721007, 201715907, 2017160107), IVATS National Key R \& D Program (2017YFC0907903, 2017YFC0112704) and Application, Industrialization, and Generalization of Surgical Incision Protector (2011B090400589).

\section{Footnote}

Reporting Checklist: The authors have completed the REMARK reporting checklist. Available at https://dx.doi. org/10.21037/tlcr-21-600

Data Sharing Statement: Available at https://dx.doi. org/10.21037/tlcr-21-600 
Peer Review File: Available at https://dx.doi.org/10.21037/ tlcr-21-600

Conflicts of Interest: All authors have completed the ICMJE uniform disclosure form (available at https://dx.doi. org/10.21037/tlcr-21-600). WL serves as an unpaid editorial board member of Translational Lung Cancer Research. The other authors have no conflicts of interest to declare.

Ethical Statement: The authors are accountable for all aspects of the work in ensuring that questions related to the accuracy or integrity of any part of the work are appropriately investigated and resolved. All procedures performed in this study were in accordance with the Declaration of Helsinki (as revised in 2013). The study was approved by the Institutional Review Board of the First Affiliated Hospital of Guangzhou Medical University (No. KLS-2020) and individual consent for this retrospective analysis was waived.

Open Access Statement: This is an Open Access article distributed in accordance with the Creative Commons Attribution-NonCommercial-NoDerivs 4.0 International License (CC BY-NC-ND 4.0), which permits the noncommercial replication and distribution of the article with the strict proviso that no changes or edits are made and the original work is properly cited (including links to both the formal publication through the relevant DOI and the license). See: https://creativecommons.org/licenses/by-nc-nd/4.0/.

\section{References}

1. Sung H, Ferlay J, Siegel RL, et al. Global Cancer Statistics 2020: GLOBOCAN Estimates of Incidence and Mortality Worldwide for 36 Cancers in 185 Countries. CA Cancer J Clin 2021;71:209-49.

2. Torre LA, Bray F, Siegel RL, et al. Global cancer statistics, 2012. CA Cancer J Clin 2015;65:87-108.

3. Shaw AT, Ou SH, Bang YJ, et al. Crizotinib in ROS1rearranged non-small-cell lung cancer. N Engl J Med 2014;371:1963-71.

4. Kwak EL, Bang YJ, Camidge DR, et al. Anaplastic lymphoma kinase inhibition in non-small-cell lung cancer. N Engl J Med 2010;363:1693-703.

5. Paez JG, Jänne PA, Lee JC, et al. EGFR mutations in lung cancer: correlation with clinical response to gefitinib therapy. Science 2004;304:1497-500.

6. Ettinger DS, Wood DE, Aisner DL, et al. NCCN
Guidelines Insights: Non-Small Cell Lung Cancer, Version 2.2021. J Natl Compr Canc Netw 2021;19:254-66.

7. Adderley H, Blackhall FH, Lindsay CR. Toxicity with small molecule and immunotherapy combinations in nonsmall cell lung cancer. Cancer Immunol Immunother 2021;70:589-95.

8. Krause DS, Van Etten RA. Tyrosine kinases as targets for cancer therapy. N Engl J Med 2005;353:172-87.

9. Chu CY, Choi J, Eaby-Sandy B, et al. Osimertinib: A Novel Dermatologic Adverse Event Profile in Patients with Lung Cancer. Oncologist 2018;23:891-9.

10. Escudier B, Eisen T, Stadler WM, et al. Sorafenib in advanced clear-cell renal-cell carcinoma. N Engl J Med 2007;356:125-34.

11. Motzer RJ, Hutson TE, Tomczak P, et al. Sunitinib versus interferon alfa in metastatic renal-cell carcinoma. $\mathrm{N}$ Engl J Med 2007;356:115-24.

12. Kerkela R, Woulfe KC, Durand JB, et al. Sunitinibinduced cardiotoxicity is mediated by off-target inhibition of AMP-activated protein kinase. Clin Transl Sci 2009;2:15-25.

13. Kerkelä R, Grazette L, Yacobi R, et al. Cardiotoxicity of the cancer therapeutic agent imatinib mesylate. Nat Med 2006;12:908-16.

14. Adenis A, Bouché O, Bertucci F, et al. Serum creatine kinase increase in patients treated with tyrosine kinase inhibitors for solid tumors. Med Oncol 2012;29:3003-8.

15. Pan H, Xia K, Zhou W, et al. Low serum creatine kinase levels in breast cancer patients: a case-control study. PLoS One 2013;8:e62112.

16. Onda T, Uzawa K, Endo Y, et al. Ubiquitous mitochondrial creatine kinase downregulated in oral squamous cell carcinoma. Br J Cancer 2006;94:698-709.

17. Camp RL, Dolled-Filhart M, Rimm DL. X-tile: a new bio-informatics tool for biomarker assessment and outcome-based cut-point optimization. Clin Cancer Res 2004;10:7252-9.

18. Wallimann T, Tokarska-Schlattner M, Schlattner U. The creatine kinase system and pleiotropic effects of creatine. Amino Acids 2011;40:1271-96.

19. Wallimann T, Wyss M, Brdiczka D, et al. Intracellular compartmentation, structure and function of creatine kinase isoenzymes in tissues with high and fluctuating energy demands: the 'phosphocreatine circuit' for cellular energy homeostasis. Biochem J 1992;281:21-40.

20. Patra S, Bera S, SinhaRoy S, et al. Progressive decrease of phosphocreatine, creatine and creatine kinase in skeletal muscle upon transformation to sarcoma. FEBS J 
2008;275:3236-47.

21. Zhang Y, Li H, Wang X, et al. Regulation of T cell development and activation by creatine kinase B. PLoS One 2009;4:e5000.

22. Hunter G, Voll C, Robinson CA. Autoimmune inflammatory myopathy after treatment with ipilimumab. Can J Neurol Sci 2009;36:518-20.

23. Yoshioka M, Kambe N, Yamamoto Y, et al. Case of respiratory discomfort due to myositis after administration of nivolumab. J Dermatol 2015;42:1008-9.

24. Sheik Ali S, Goddard AL, Luke JJ, et al. Drug-associated dermatomyositis following ipilimumab therapy: a novel immune-mediated adverse event associated with cytotoxic T-lymphocyte antigen 4 blockade. JAMA Dermatol 2015;151:195-9.

Cite this article as: Jiang Y, Su Z, Lin Y, Xiong Y, Li C, Li J, Wang R, Zhong R, Cheng B, He J, Xie Z, Liang W. Prognostic and predictive impact of creatine kinase level in non-small cell lung cancer treated with tyrosine kinase inhibitors. Transl Lung Cancer Res 2021;10(9):3771-3781. doi: 10.21037/tlcr-21-600
25. Hua C, Boussemart L, Mateus C, et al. Association of Vitiligo With Tumor Response in Patients With Metastatic Melanoma Treated With Pembrolizumab. JAMA Dermatol 2016;152:45-51.

26. Sanlorenzo M, Vujic I, Daud A, et al. Pembrolizumab Cutaneous Adverse Events and Their Association With Disease Progression. JAMA Dermatol 2015;151:1206-12.

27. Lechner MG, Vyas CM, Hamnvik OR, et al. Hypothyroidism During Tyrosine Kinase Inhibitor Therapy Is Associated with Longer Survival in Patients with Advanced Nonthyroidal Cancers. Thyroid 2018;28:445-53.

28. Villadolid J, Amin A. Immune checkpoint inhibitors in clinical practice: update on management of immunerelated toxicities. Transl Lung Cancer Res 2015;4:560-75. 


\section{Supplementary}

A

Strata + Group=1 + Group=2

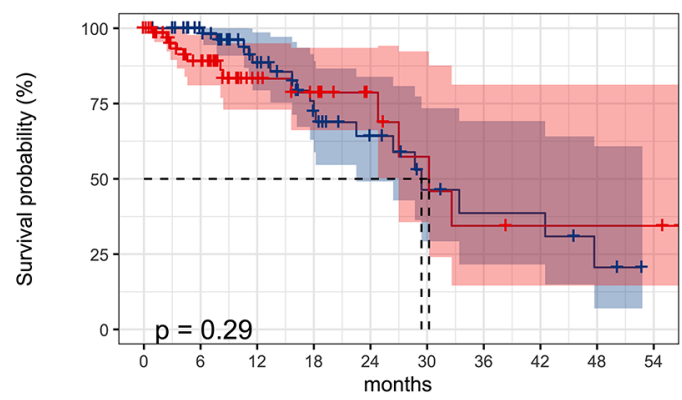

Number at risk
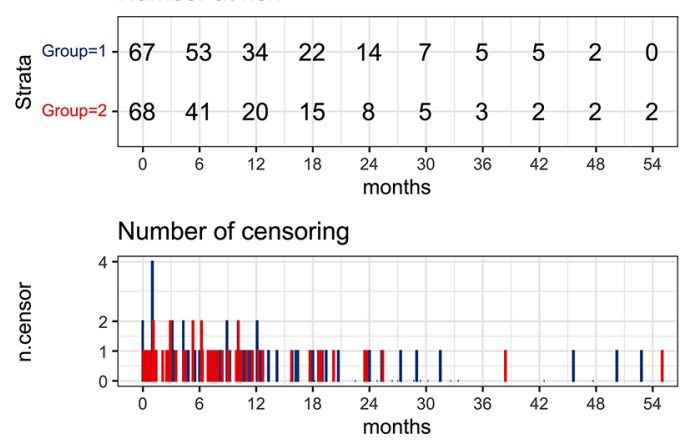

B
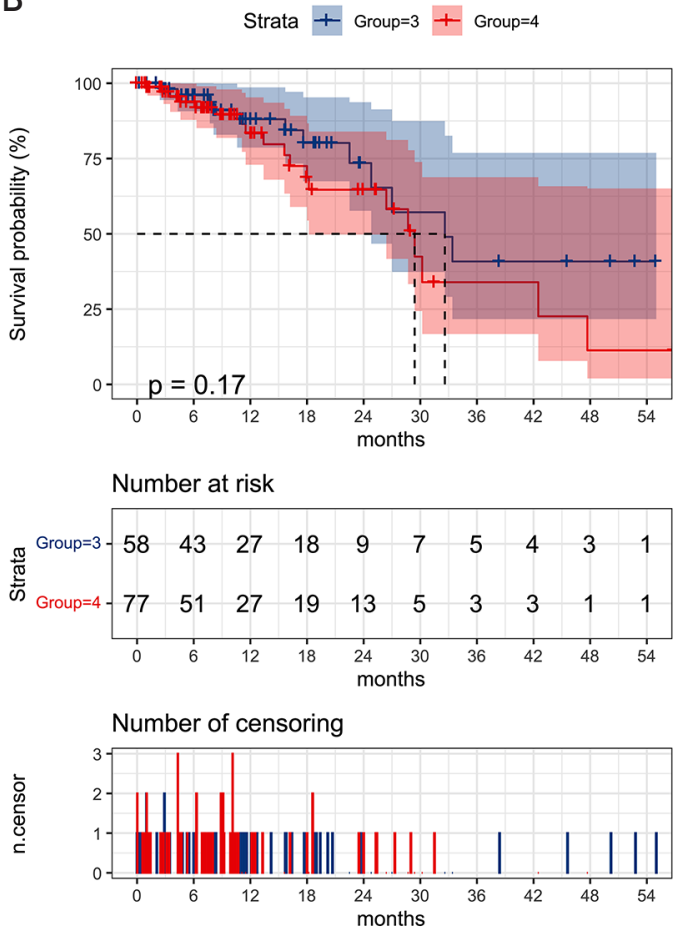

Figure S1 Kaplan-Meier curves for overall survival in advanced NSCLC patients treated with TKIs, stratified by (A) baseline CK level, and (B) CK change during TKI therapy. CK, creatine kinase; NSCLC, non-small cell lung cancer; TKI, tyrosine kinase inhibitor. 


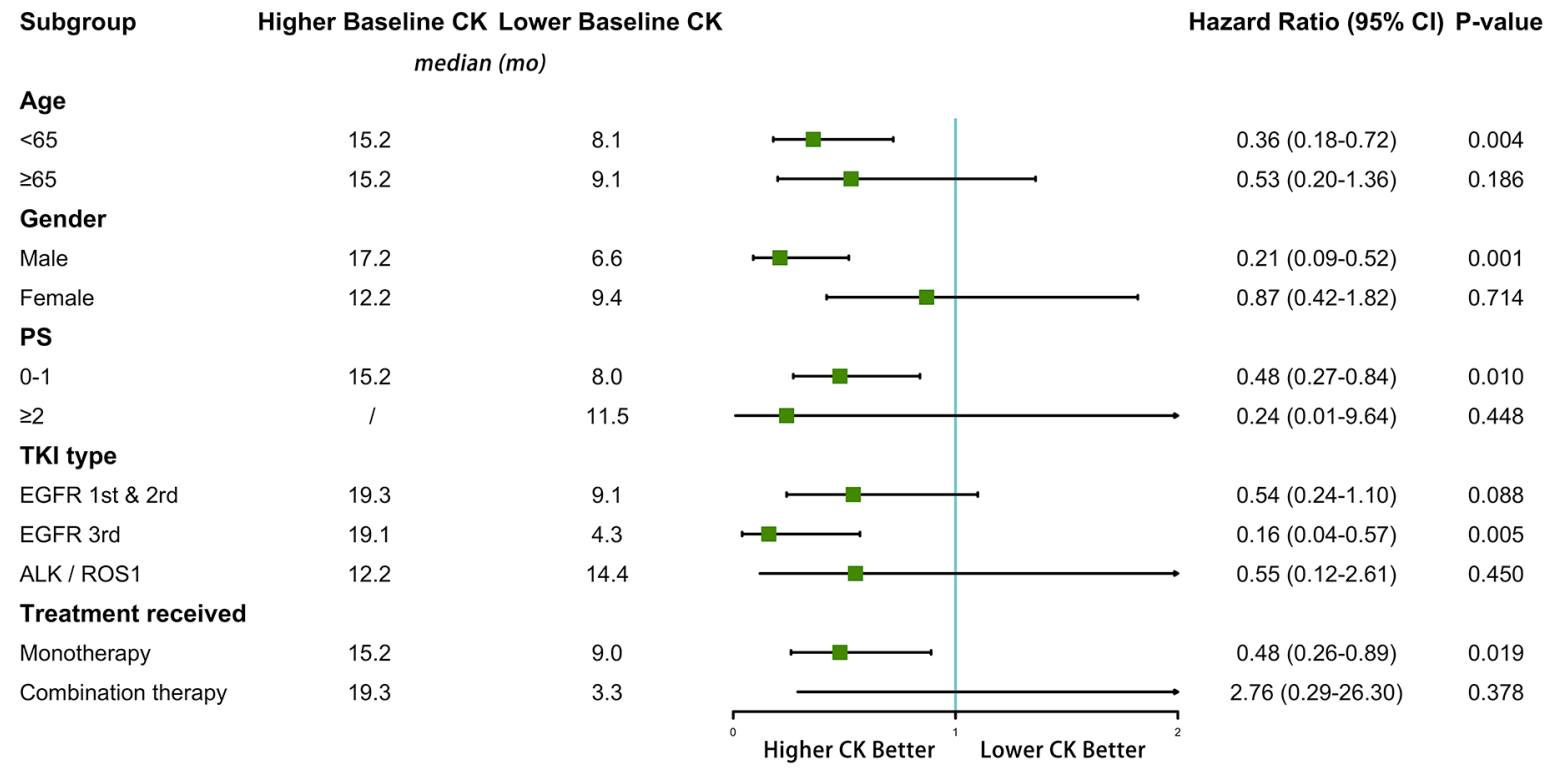

Figure S2 Subgroup analyses of the effect of baseline CK level on TKI efficacy. Progression-free survival and subgroup analyses were estimated by the Kaplan-Meier method and Cox proportional-hazards model. Covariables included age, gender, PS, TKI type, and treatment received. ALK, anaplastic lymphoma kinase; CI, confidence interval; CK, creatine kinase; EGFR, epidermal growth factor receptor; PS, performance status; ROS-1, c-ROS oncogene 1; TKI, tyrosine kinase inhibitor.

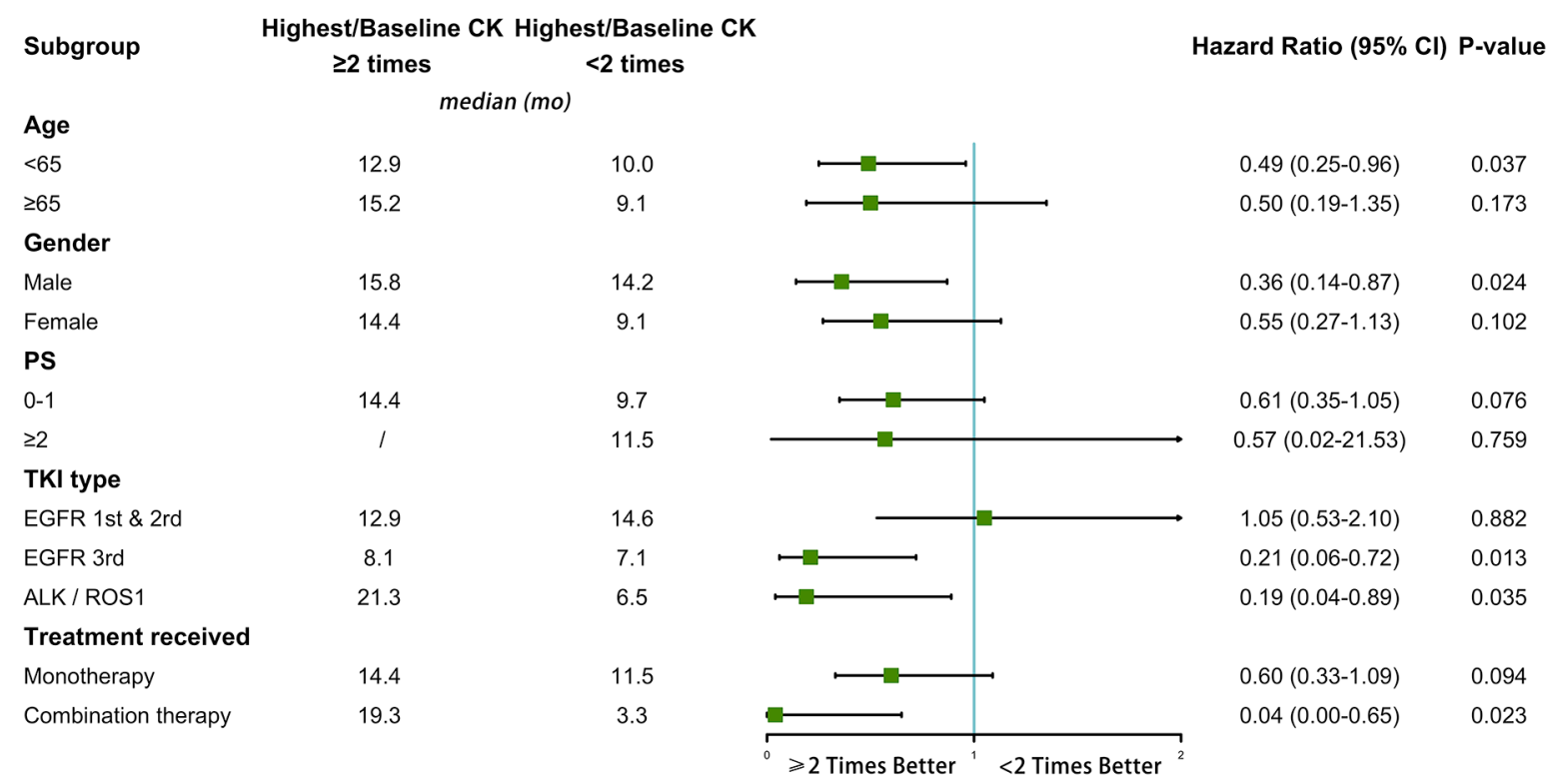

Figure S3 Subgroup analyses of the effect of CK elevation on TKI efficacy. Progression-free survival and subgroup analyses were estimated by the Kaplan-Meier method and Cox proportional-hazards model. Covariables included age, gender, PS, TKI type, and treatment received. ALK, anaplastic lymphoma kinase; CI, confidence interval; CK, creatine kinase; EGFR, epidermal growth factor receptor; PS, performance status; ROS-1, c-ROS oncogene 1; TKI, tyrosine kinase inhibitor. 